\title{
Application of IR thermography for thermal inverse modelling to evaluate the local power loss in punched ferromagnetic strips
}

\author{
by M. Strąkowska*, Z. Gmyrek ${ }^{* *}$ and B. Więcek
}

*Lodz University of Technology, Institute of Electronic, 90-924, Wólczańska 211/215, Łódź, Poland, wiecek@p.ldz.pl

"Lodz University of Technology, Institute of Mechatronics and Information Systems, 90-924, Stefanowskiego 18/22, Łódź, Poland, zbigniew.gmyrek@p.lodz.pl

\section{Abstract}

The authors deal with the problem of determining the local properties of ferromagnetic material subjected to mechanical cutting. They use a IR camera to record local temperature rise in the punched region. Next, the temperature measured is used in the numerical models that allow estimation of the power loss density distribution. A simple inverse thermal model for a ferromagnetic strip with non-uniform power loss are presented. The correctness of the results obtained from the simple inverse model is verified using the 3D thermal finite element model (FEM) and compared with the measurement results.

\section{Introduction}

It is known that the mechanical cutting changes the local properties of ferromagnetic materials, located close to the cut edge. The changes are the result of additional internal stresses in the material structure, as well as breaking of grains and magnetic domain structures. This phenomenon has been under investigation for many years, using invasive and non-invasive measurement methods. The invasive methods include, for example, the method of measuring coils placed in drilled holes [1]. Unfortunately, this method change the local properties of the material. In contrast, there are noninvasive methods, such as, for example, magnetovision one [2] and method based on a neutron grating interferometry [3], which do not vary the local properties of the material. The scientists also use methods that are partially invasive ones, e.g. the needle probe method [4] (measuring needles absorb a portion of the heat generated in the area) or microhardness measurement method [5] (introducing additional microstrains in the structure). The authors of this paper show that it is possible to use IR camera to record the local temperature rise in the punched area, and then by using the analytical or/and numerical thermal modeling can determine the density distribution of power loss in the zone near the cut edge of the ferromagnetic strip [6]. Two mathematical heat transfer models describing the dynamic thermal problem in a ferromagnetic strip were used to find the distribution of heat generation in a strip. The temperature fields calculated by these models have been compared with the measurement results.

\section{Numerical models for the analysis of the loss density distribution}

At first, the authors implemented 3D numerical model to estimate the power loss density distributions -fig. 1.The 3D thermal model was built in the OPERA commercial software package. The appropriate boundary conditions and thermal parameters of the ferromagnetic strip were chosen as it shown in fig. 1 . The cut edge is located on the front side $(0 z y$ plane on the left side of the model with the heat transfer coefficient $h=5 \mathrm{~W} / \mathrm{m}^{2} \mathrm{~K}$ ).

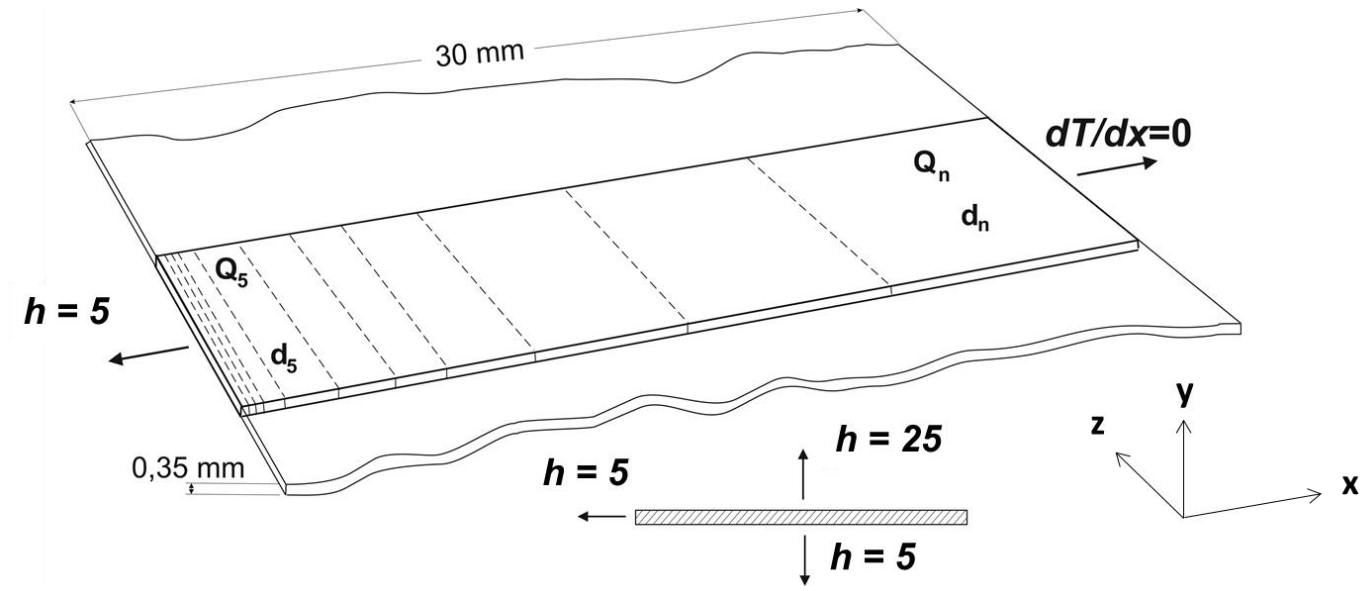

Fig. 1. Sectional model of a magnetic strip used in both numerical and analytical modeling (heat transfer coefficients in $\mathrm{W} / \mathrm{m}^{2} \mathrm{~K}$ ) 


\subsection{1/qirt.2016.150}

Assuming the axial symmetry of heat propagation in a ferromagnetic strip subjected to cut allows significant acceleration of computing time. The model defines several zones (sections) where the different power is dissipated. Modeling of the magnetic strip's sections requires the proper boundary and interface conditions for the FEM modeling. The boundary conditions mentioned below were applied.

- Right and left sides of the model - symmetry was assumed because the physical phenomenon has the same nature along the cut surface, $T\left(z_{\min }\right)=T\left(z_{\max }\right)-$ fig. 1 .

- Front and back sides - heat is removed from the surfaces. The heat transfer coefficient on the front surface $(x=0)$ was assumed to be equal to $5 \mathrm{~W} / \mathrm{m}^{2} \mathrm{~K}$ while on the back side $(x=0.03 \mathrm{~m})$ the adiabatic boundary condition is taken.

- Bottom side - heat is removed from this surface. The heat transfer coefficient was assumed to be equal to $5 \mathrm{~W} / \mathrm{m}^{2} \mathrm{~K}$ (bottom side is for $y=0$ ),

- Upper side - heat is removed from this surface. The heat transfer coefficient was assumed to be equal to $25 \mathrm{~W} / \mathrm{m}^{2} \mathrm{~K}$ (upper side is for $y=0.0035 \mathrm{~m}$ ).

The OPERA 3D software describes three dimensional thermal fields which can be represented using a single scalar potential $T$. Physically, $T$ is the usual temperature field. The thermal gradient intensity $D T$ is given by the equation (1).

$$
D T=-\nabla T
$$

and the heat flux density $q$ by (2).

$$
q=\lambda D T
$$

where $\lambda$ is the thermal conductivity. Energy conservation requires the time rate of the change of internal thermal energy within a volume to be equal to the power density minus the change of the heat flux through its surface. Applying the energy conservation to an infinitesimal volume yields

$$
\rho c_{w} \frac{\partial}{\partial t} T=p_{v}-\nabla \cdot q
$$

where $p_{v}$ is the volumetric heat source density, $\rho$ the mass density, and $c_{w}$ the specific heat of the magnetic material. Combining above mentioned equations it is possible to write the well-known diffusion equation for the temperature distribution.

$$
\rho c_{w} \frac{\partial}{\partial t} T-\nabla \cdot \lambda \nabla T=p_{v}
$$

The ferromagnetic strip has following dimensions: $t_{s}=350 \mu \mathrm{m}$ thick, $d=30 \mathrm{~mm}$ width, $l=300 \mathrm{~mm}$ length, whereas the modeled piece was $5 \mathrm{~mm}$ long (this is the distance between right and left sides of the model). The model was divided into 10 sections with dimensions listed in table 1. It was assumed that the heat is dissipated to the ambient by convection and radiation according to the estimated convective heat transfer coefficients. The authors adapted in each section evenly distributed heat source, whose values were sought.

Table 1. Dimensions along $X$ axis, for all 10 sections the model was divided into (in $\mathrm{mm}$ )

\begin{tabular}{|c|c|c|c|c|c|c|c|c|c|}
\hline$d_{1}$ & $d_{2}$ & $d_{3}$ & $d_{4}$ & $d_{5}$ & $d_{6}$ & $d_{7}$ & $d_{8}$ & $d_{9}$ & $d_{10}$ \\
\hline 0.5 & 1 & 2 & 2.5 & 3 & 4 & 4 & 4 & 4 & 5 \\
\hline
\end{tabular}

The model is placed horizontally and therefore the heat transfer coefficient has two different values for the bottom and top sides of the sample. In the 3D model, the following parameters, described in table 2, were used. Authors made the assumption, that parameters are independent from the temperature.

Table 2. Values of thermal parameters used in the model

\begin{tabular}{|c|c|c|c|}
\hline Density $\rho, \mathrm{kg} / \mathrm{m}^{3}$ & $\begin{array}{c}\text { Thermal conductivity } \lambda, \\
\mathrm{W} / \mathrm{m} \cdot \mathrm{K}\end{array}$ & Specific heat $\mathrm{c}_{\mathrm{w}}, \mathrm{J} / \mathrm{kgK}$ & $\begin{array}{c}\text { Convective heat transfer } \\
\text { coefficient } \mathrm{h}, \mathrm{W} / \mathrm{m}^{2} \mathrm{~K}\end{array}$ \\
\hline 7850 & 44.1 & 475 & 25.1 \\
\hline
\end{tabular}

\section{Analytical 1D thermal models in frequency domain}

A simple, 2D analytical model was implemented in MATLAB environment. Due to the symmetry of heat transfer along $O z$ axis and relatively low value of the thickness comparing to other dimensions, the presented model was reduced into 1D one. The 3D FEM model, presented above, was used to validate the results of 1D simulations. In order to calculate the temperature analytically, 1D thermal model in was defined in Laplace domain. The strip of $30 \mathrm{~mm}$ long was divided 


\subsection{1/qirt.2016.150}

into 11 rectangular sectors. It was similarly as for numerical model, but instead of 10 , now we use 11 sections - fig. 1 . The thickness $t_{s}$ of the strip was much lower than its length $d$ and the magnetic field was uniformly distributed along the strip width. In consequence 1-D thermal model was used in simulations. 1-D thermal model (5) in Laplace transform domain includes convection cooling along the strip. Convection was modelled using heat transfer coefficient $h_{t}=h_{1}+h_{2}$ which was constant but different for both sides of the strip. The model takes a form [6]

$$
\begin{aligned}
& \lambda \frac{d^{2} T}{d x^{2}}-\left(\rho \cdot c_{s} \cdot s+\frac{\alpha}{t_{s}}\right) T=-p_{v i} \\
& \frac{d^{2} T}{d x^{2}}-\frac{T}{L^{2}}=-\frac{p_{v i}}{\lambda}
\end{aligned}
$$

where $\rho$ is material density, $c_{s}$ is specific heat, $\lambda$ is thermal conductivity coefficient, $t_{s}$ is thickness of a strip, $p_{v i}$ is the volumetric power density dissipated in each section, $i=1 . .11$. Symbol $L$ denotes so-called diffusion length and it is a complex value given by eqn. (6).

$$
L(s)=\frac{1}{\sqrt{\frac{\rho \cdot c_{s} \cdot s}{\lambda}+\frac{h_{t}}{\lambda t_{s}}}}
$$

where $h$ is heat transfer coefficients $h_{t}=h_{1}+h_{2}$

The solution of eqn. (5) for each section is analytical, expressed by eqn. (7).

$$
T_{i}(s)=A_{i}(s) \cdot e^{-\frac{x}{L(s)}}+B_{i}(s) \cdot e^{\frac{x}{L(s)}}+\frac{p_{v i} L^{2}}{2 \lambda}
$$

The input flux with convection coefficient $h$ is defined as:

$$
-\left.\lambda \frac{d T_{1}}{d x}\right|_{x=0+}=-\left.h \cdot T_{1}\right|_{x=0-}
$$

where $T_{1}$ is the temperature if the first section - fig. 1. At the end of the strip, we neglect the cooling.

$$
\left.\frac{d T_{11}}{d x}\right|_{x=d 11-}=0
$$

where $T_{11}$ is the temperature if the last $11^{\text {th }}$ section - fig. 1 .

The interface between the neighbor sections is described using the conditions of continuous temperature and heat flux eqn. (10).

$$
\begin{aligned}
& \left.\frac{d T_{n}}{d x}\right|_{x=d n-}=\left.\frac{d T_{n+1}}{d x}\right|_{x=0+} \\
& \left.T_{n}\right|_{x=d n-}=\left.T_{n+1}\right|_{x=0+}
\end{aligned}
$$

Equations (9) and (10) constitute the set of 22 linear equations where $A_{i}$ and $B_{i}(i=1 . .11)$ are the unknown integration constants. Such set of linear equations can be presented as:

$$
Y=Z * X
$$

where the matrix $\boldsymbol{Z}_{22 \times 22}$ and $\boldsymbol{Y}_{1 \times 22}$ take the form presented below. 


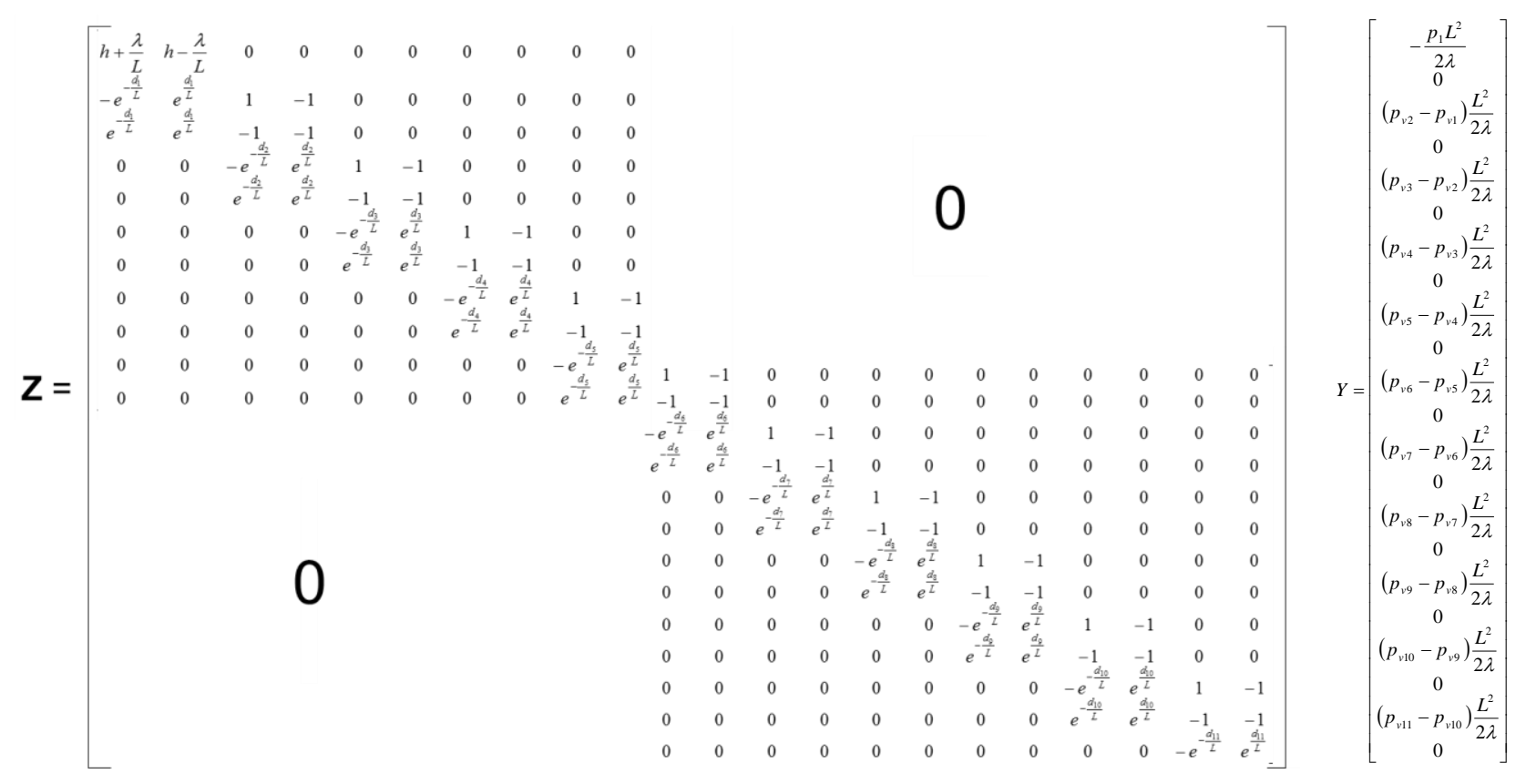

Matrix $X$ consisting of unknown integration constants $X=\left[\begin{array}{c}A_{i} \\ B_{i}\end{array}\right]$ can be calculated numerically by matrix inversion or by using e.g. Gaussian elimination method.

$$
X=Z^{-1} * Y
$$

Finally, one can calculate temperature in any point in each section of the ferromagnetic strip.

For analytical modeling one divided the strip into 11 sections of the sizes presented in Table 3 .

Table 3. Dimensions in $\mathrm{m}$ for all 11 sections a sample (in $\mathrm{mm}$ )

\begin{tabular}{|c|c|c|c|c|c|c|c|c|c|c|c|}
\hline$d_{1}$ & $d_{2}$ & $d_{3}$ & $d_{4}$ & $d_{5}$ & $d_{6}$ & $d_{7}$ & $d_{8}$ & $d_{9}$ & $d_{10}$ & $d_{11}$ \\
\hline 0.33 & 0.33 & 0.34 & 0.5 & 1 & 1 & 1 & 2.5 & 6 & 8 & 9 \\
\hline
\end{tabular}

\section{Inverse thermal model}

The inverse thermal modeling was implemented in the way presented in fig. 2. First we measure the temperature distribution on a strip during heating up process, after the powering of the sample by applying the magnetic flux into it. The heating is non-uniform due to the deterioration of the edge of a material. Then the Laplace transform is applied to present temperature in frequency domain. In parallel, the analytical model (5-12) is calculated. It gives directly the Nyquist plot of temperature calculated in different points of a magnetic strip. Finally, the optimization is applied to adjust the power $P_{i}$ in all sections in a strip to minimize the difference between the temperature from the model and measurement in frequency domain for arbitrary chosen points on the strip. 


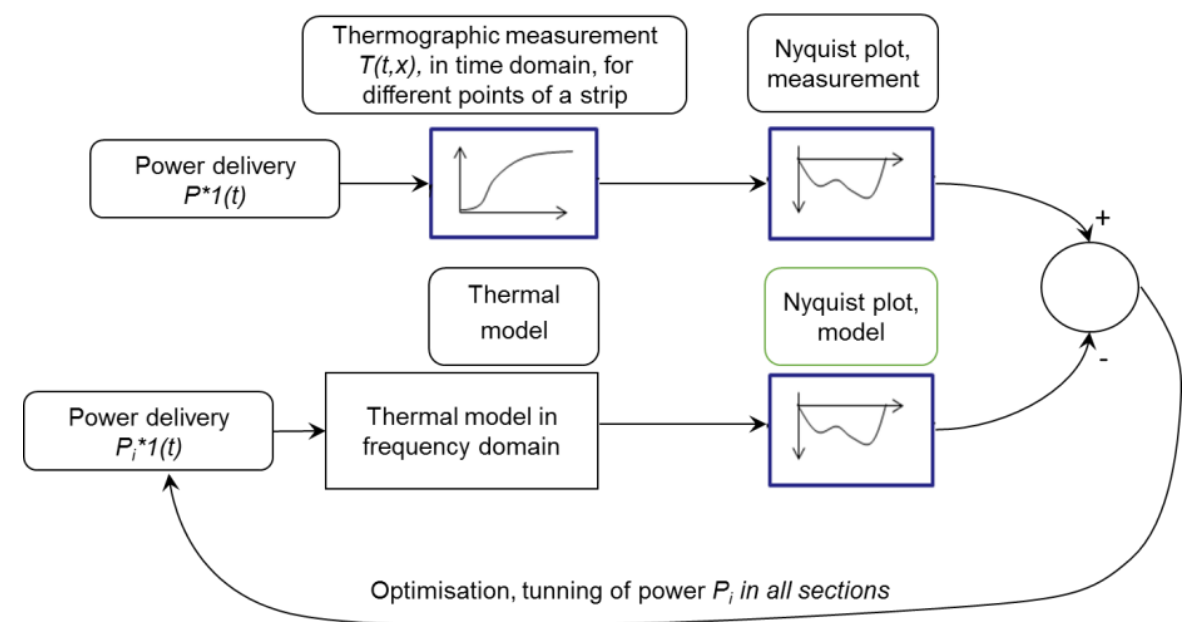

Fig. 2. Inverse thermal model of a ferromagnetic strip to get the power loss distribution

Thermal inverse modeling is implemented in Matlab ${ }^{\circledR}$ environment using in-built optimization methods. Two methods were tested - patternsearch and fminsearch. Function fminsearch is based on Nelder-Mead algorithm (so called downhill simplex method). It is one of the nonlinear unconstraint optimization technique used for the multivariable aim functions. The simplex method searches a local minimum of this function and it is recommended when the derivatives are not known and no constraints are needed [1, 2]. Patternsearch is known as direct-search, derivative-free or black box method. This method is an heuristic one when no derivative of the function is known. In contrast to fminsearch method, it can be implemented with the arbitrary defined bounds [3]. This method implements two directions of searching an optimum: exploratory and pattern ones. The first approach improves the direction of the movement, while the second one lengthen the moves as long as the improvement continues. After some trials, we decided to use patternsearch method.

\section{Results}

Thermographic measurements and modeling were performed for 3-cm ferromagnetic strip divided into 10/11 sections as it was mentioned above. The thermal images recorded for different moments in time are presented in fig. 3 . One can notice the temperature in the punched area at the edge (at the bottom) is slightly higher than elsewhere. In addition, fig. 3 presents the arbitrary points, the temperature values were taken from for the optimization. The exemplary temperature variation in time obtained from the thermal model for the given power densities in all 11 sections of the strip is shown in fig. 4. The curves plotted by dotted lines are the results of the measurement.
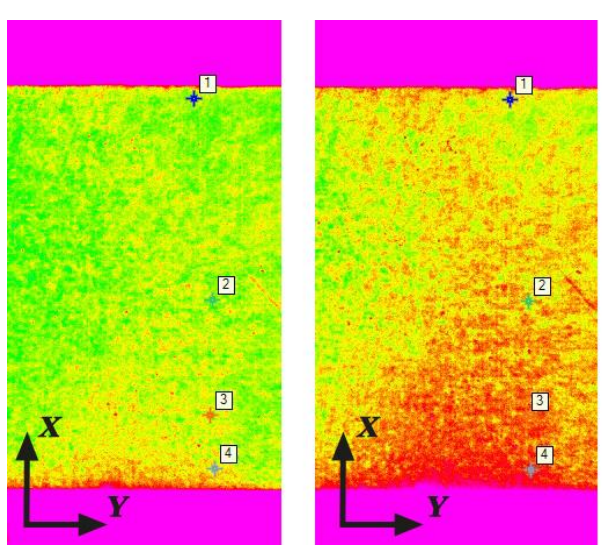

a)

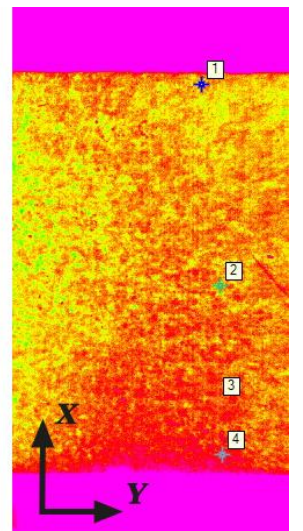

4.

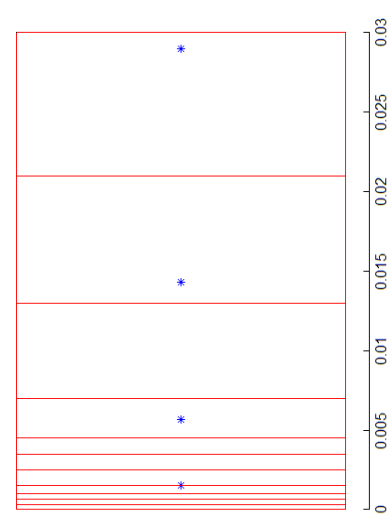

b)

Fig. 3. Thermograms of the ferromagnetic strip for different moments in time (a) and the points indicated and used for optimization 


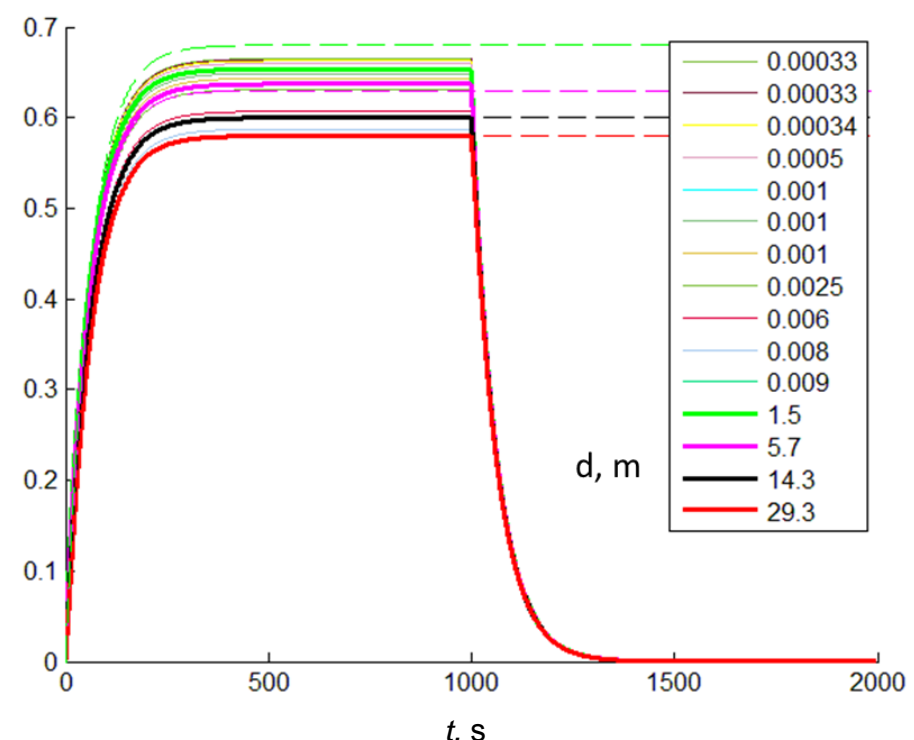

Fig. 4. Temperature change (in ${ }^{\circ} \mathrm{C}$ ) during heating up (and cooling) with the values obtained from the thermal camera measurement for different places on the strip

At the beginning we were trying to compare the temperature in the arbitrary chosen points to adjust the power density distribution in the strip. It did not give the satisfactory results, that were already published the literature [1-6]. We were trying the different sets of points located in different places on the strip. One could notice that it was rather difficult to find the appropriate number and positions of such points. The results obtained were worse or better without any reasonable conclusion. Finally, one decided to assume the shape of the power loss distribution in a sample. Among few approximation candidates considered, the exponential one (13) allowed to get the best results.

$$
f(x)=\left\{\begin{array}{ccc}
A e^{-\frac{x}{L}}+B & \text { for } & x>0.001 \\
A e^{-\frac{0.001}{L}}+B & \text { for } & x \leq 0.001
\end{array}\right.
$$

The power density distribution in the punched ferromagnetic strip was obtained using the proposed approach as presented in fig. 5. It agrees with the results published in the literature and obtained using other methods [1-3]. Figure 6 shows the screen of the program for optimization and calculating the model's parameters. First graph (on the left) shows the Nyquist plot of the temperature while the second one presents in temperature vs. time after inverse Laplace transformation. The model's parameters were set before starting the program. Thermal parameters and the distances were known and the values of power densities were estimated according to the function (13). Finally, we plot the power density distribution using both numerical and analytical modeling - fig. 5 . 


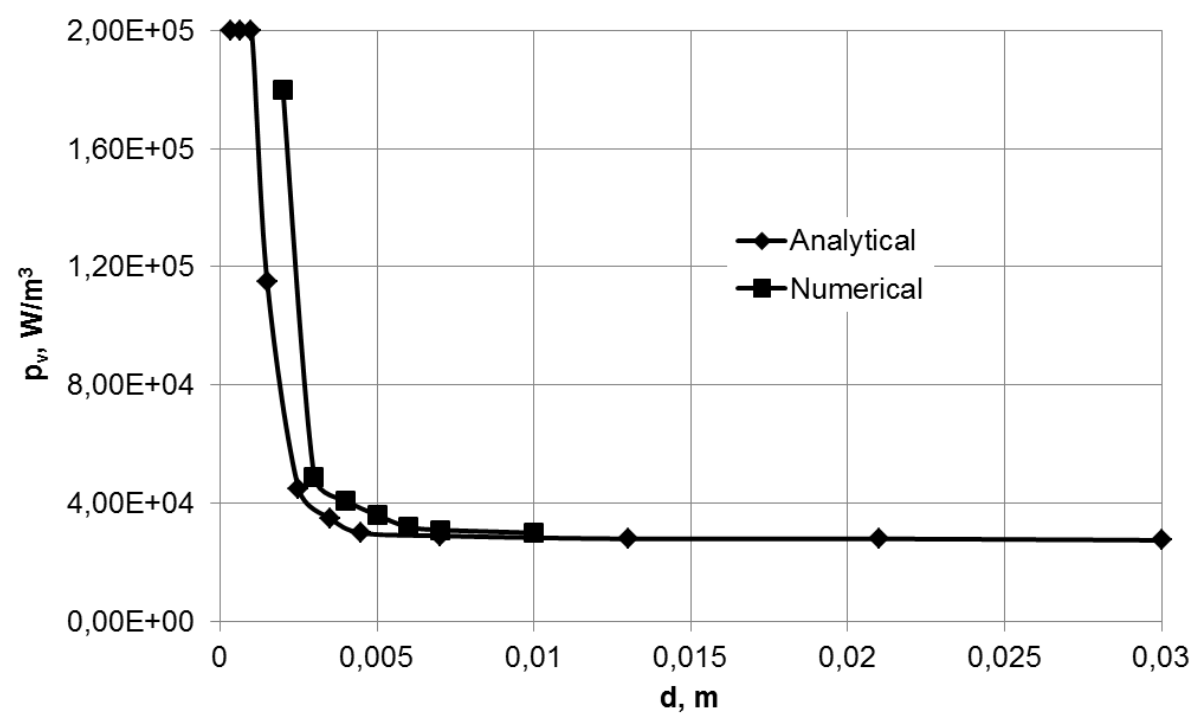

Fig. 5. Power density distribution on a punched ferromagnetic strip calculated by analytical and numerical model

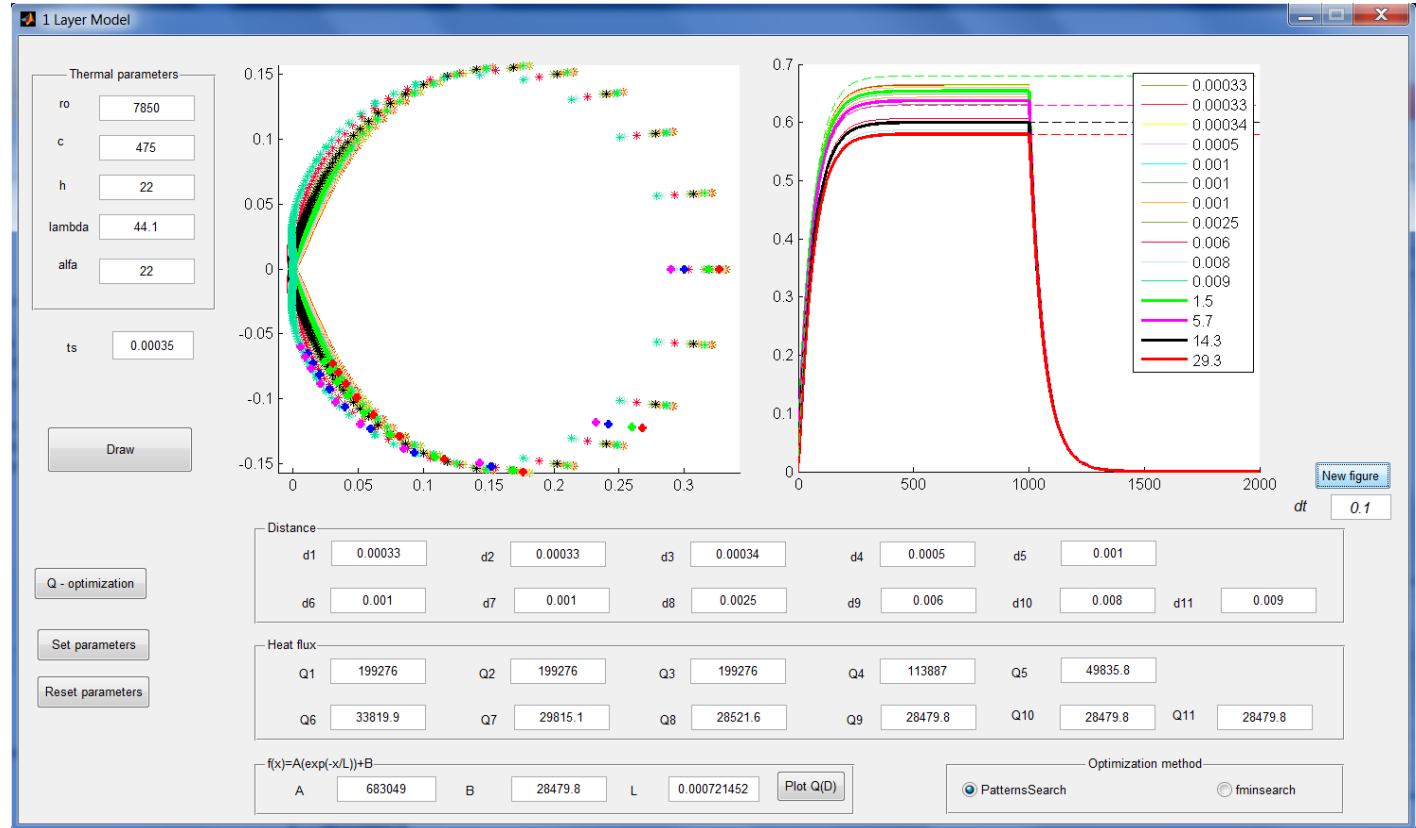

Fig. 6. Screen of the program for optimization and estimation the power density distribution in the ferromagnetic strip

\section{Conclusions}

The authors dealt with the solution of the inverse problem, namely to find the distribution and the power density of the heat sources, existing in the magnetically anisotropic strip subjected to the cutting process. During measurements, the authors enforced relatively high flux density value, in order to obtain the same magnetic flux penetrating the material portions lying close and far from the cut edge. Thus, in this way we induced non-uniform heating of the test strip. The results obtained using the analytical method and FEM model are similar but not identical, what results from the need for a uniform distribution of heat sources in the strip segment. The resulting ratio of the heat density of heat sources located in close proximity to the edge, to the power density of heat sources in the remote parts of the strip is similar to the measurement results reported in the literature. The main advantage of the analytical method is its rapidity, although the authors are aware of the need to "tune" this model.

\section{REFERENCES}

[1] Peksoz A., Erdem S., Derebasi N., "Mathematical model for cutting effect on magnetic flux distribution near the cut edge of non-oriented electrical steels", Computational Materials Science, 43 (2008), 1066-1068 
[2] Baudouin P., Houbaert Y., Tumanski S., "Magnetic local investigations of non-oriented electrical steels after tensile deformation", Journal of Magnetism and Magnetic Materials, 254-255 (2003), 32-35

[3] Sieberg R., Wetzig A., Beyer E., Betz B., Grunzweig Ch., Lehmann E., "Localized investigation of magnetic bulk property deterioration of electrical steel“, Proc. of International Electric Drives Production Conference, 2013

[4] Loisos G., Moses A. J., "Demonstration of a new method for magnetic flux measurement in the interior of a magnetic material", Sensors and Actuators A, 106 (2003), 104-107

[5] Kurosaki Y., Mogi H., Fujii H., Kubota T., Shizoaki M., "Importance of punching and workability in non-oriented electrical steel sheets", Journal of Magnetism and Magnetic Materials, 320 (2008), 2474-2480

[6] Gmyrek Z., Strąkowska M., Więcek B., "A method of local magnetic loss determination in punched ferromagnetic strip", Journal of Magnetism and Magnetic Materials, 355 (2014), 282-288

[7] John A. Nalder, Roger Mead. A Simplex Method for Function Minimization. „The Computer Journal”. 7 (4), s. 308-313, 1965. DOI: 10.1093/comjnl/7.4.308.

[8] Lagarias, J.C., J. A. Reeds, M. H. Wright, and P. E. Wright, "Convergence Properties of the Nelder-Mead Simplex Method in Low Dimensions," SIAM Journal of Optimization, Vol. 9 Number 1, pp. 112-147, 1998.

[9] Hooke, R.; Jeeves, T.A. (1961). "Direct search" solution of numerical and statistical problems". Journal of the Association for Computing Machinery (ACM) 8 (2): 212-229. doi:10.1145/321062.321069.

[10] John W. Chinneck, Practical Optimization: A Gentle Introduction, Canada 2014, http://www.sce.carleton.ca/faculty/chinneck/po.html 